Moroccan J. of Pure and Appl. Anal. (MJPAA)

Volume 5(2), 2019, Pages 186-196

ISSN: Online 2351-8227 - Print 2605-6364

DOI 10.2478/mjpaa-2019-0014

\title{
Modeling Tuberculosis Among Healthcare Workers
}

\author{
T.S. FANiran ${ }^{1, a}$, A.O. FAlAdE ${ }^{2, b}$ and T.O. Alakija ${ }^{3, c}$
}

\begin{abstract}
Aвstract. A mathematical model for transmission dynamics of tuberculosis among healthcare workers is formulated. Tuberculosis is an airborne disease caused by Mycobacterium tuberculosis bacteria that affect the lungs of a host. Previous research had concentrated on mathematical modeling of transmission dynamics of tuberculosis without considering the impact of compliance rate to particulate respirator by healthcare workers on the transmission. Therefore, how compliance rate to particulate respirator reduces the transmission of tuberculosis is an active question, and we develop a new system of ordinary differential equations that explicitly explores the impact of compliance rate to particulate respirator by healthcare workers upon transmission. Rigorous analysis of the model shows that the disease-free equilibrium point is locally asymptotically stable when the basic reproduction number, $R_{o}<1$. This is established through the analysis of characteristic equation. Basic reproduction, $R_{o}$ is the number of new cases that an existing case generates on average over the infectious period in a susceptible population. We also show that the endemic equilibrium point is locally asymptotically stable for $R_{o}>1$, by using Routh-Hurwitz criteria for stability . Sensitivity analysis is carried out to determine the relative importance of the model parameters to the disease transmission. The result of the sensitivity analysis shows that the most sensitive parameter is $\beta$ (Human-to-human transmission rate), followed by $\Lambda$ (Human recruitment rate). Also, the result shows that increase in $\psi$ (compliance rate to particulate respirator by healthcare workers) leads to decrease in $R_{o}$ which reduces tuberculosis spread among healthcare workers.
\end{abstract}

2010 Mathematics Subject Classification.34C20 22E30 92D30.

Key words and phrases. Tuberculosis; Respirator; Basic Reproduction Number; Stability; Sensitivity Analysis.

Received April 27, 2019 - Accepted November 28, 2019.

(C)The Author(s) 2017. This article is published with open access by Sidi Mohamed Ben Abdallah University.

${ }^{1}$ Department of Computer Science, Lead City University, Lagos-Ibadan Expressway, Ibadan, Oyo State, Nigeria.

${ }^{2}$ Department of Mathematics, African Institute for Mathematical Sciences, Mbour, Senegal.

${ }^{3}$ Department of Statistics, Yaba College of Technology, Lagos State, Nigeria.

a e-mail: tayefaniran@yahoo.com

b e-mail: abdulahi.falade41@gmail.com

c e-mail: lawaltope2003@yahoo.com . 


\section{Introduction}

Tuberculosis is a major public health concern worldwide which causes a high rate of mortality. It is an infectious disease caused by bacteria known as Mycobacterium tuberculosis. The bacteria usually attach the lungs, but can also attack any part of the body such as kidney, spine and brain [2]. Tuberculosis is transmitted from person to person through droplets from the throat and lungs of people with the active respiratory disease, when coughing or sneezing [10]. The symptom of active TB of the lung are cough, chest pain, shortness of breast, weakness, weight loss, fever and night sweats.

Tuberculosis case increased during the year of 2013 to 2015. The case number increased up to 34 percent in India. Globally, there were 4.3 million cases at which the cases happened in India, Indonesia and Nigeria [15]. However, medication is needed when one has already been infected. The medication can generally take 6 months of time which consists of two stages; the intensive stage for 2 months and the continuation stage for 4 months [4]. The treatment is intended to cure the patients until they are fully recovered, to prevent death, recurrence and to reduce the rate of transmission.

Over the years, mathematical modeling of tuberculosis has become an important tool in understanding the transmission dynamics of the disease. It also helps to predict and control the spread of TB problem in the future. The spread of TB disease can be modelled with some types of epidemic models including the model of SIR (Susceptible, Infected, Recovered). Nainggolan et al. (2013) studied tuberculosis transmission with recurrent infection and vaccination where they obtained the non endemic and endemic fixed points. They analysed the stability of the fixed points and the relation to the basic reproduction number. Dago et al. (2015) examined stability analysis of deterministic mathematical model for transmission dynamics of tuberculosis. In their model, local stability of the disease-free and endemic equilibrium solution are established using the basic reproduction number, $R_{0}$. They also performed numerical simulations to validate their theoretical results. Taufiq et al. (2017) formulated mathematical model of tuberculosis epidemic where they considered recovery time delay since TB patients need to undergo medication with a period of time in order to recover. Their model is developed in SIR type where the population is divided into three groups, susceptible (S), infected (I) and recovered (R). They established the equilibrium points of the model as well as the stability of the fixed points. Virendra et al. (2018) proposed a mathematical model of tuberculosis with drug resistance to a first and second line of treatment. They obtained the basic reproduction number for their model using next generation matrix. They investigated the equilibrium points of the model and established the global stability of the disease-free and endemic equilibrium points. They found that when $R_{0}<1$, then the disease dies out and when $R_{o}>1$, then the disease persists. Yu et al. (2018) improved dynamic model of tuberculosis considering population dynamic and drug resistance. They analysed the feasible region, equilibrium points and stability of their model. They carried out numerical simulation of their model to predict the development of the epidemic situation and analysed the impact of model parameters on TB development. Their simulation results showed that the key to better prevent and control the spread is to improve detection rate. Leontine et al. (2019) formulated a mathematical model to assess vaccination and effective contact rate impact in the spread of tuberculosis. They carried out stability analysis proving the global asymptotic stability of disease-free and endemic equilibrium using the Lyapunov-Lassale's method. They performed numerical simulation using some TB data found in the literature in relation with Cameroon. Their results showed that vaccination coverage is not sufficient to control TB and effective contact rate has a high impact in the spread of TB.

The model proposed in this paper is unique because it incorporates the compliance rate to particulate respirator by the susceptible individuals (healthcare workers) in order to prevent TB movement in the air from patients to the healthcare workers. This is one of the tuberculosis control measures of the World Health Organization. Special protection is necessary for the healthcare workers when they are in close contact to TB patients since these healthcare workers are those who work the hardest by putting themselves in harm's way because they want to help patients. So, when a TB patient comes in, and sometimes, particulate respirators are not immediately available, then any doctor that goes in with the mind that he or she wants to help the patient, will be infected. Therefore, hospitals should see the importance in investing in particulate respirators and increase the compliance rate to particulate respirators by the healthcare workers so as to reduce TB spread. For this situation, a very specific form of protection is needed, the use of particulate respirator. A particulate respirator is the device that 
protects the healthcare workers from harmful airborne agent. When working in an environment containing TB bacteria, respirator compliance can reduce the chances of healthcare workers of contacting tuberculosis.

The remaining part of this work is organized as follows: Section 2 presents the model formulation and shows its basic property. In section 3, detailed disease-free equilibrium analysis is presented. Section 4 deals with endemic equilibrium analysis. Section 5 deals with sensitivity analysis and section 6 discusses results which concludes the modeling work.

\section{Model Formulation and its Basic Property}

The population of susceptibles is generated by the recruitment or immigration of individuals at the rate $\Lambda$. It decreases following a contact with the infectious individuals, vaccination of susceptibles, respirator compliance rate of susceptible individuals and natural death of susceptible individuals at the rates $\beta, v, \psi$ and $\mu$. Thus we have

$$
\frac{d S}{d t}=\Lambda-\beta S I-v S-\psi S-\mu S
$$

The infectious population is generated by the interaction of susceptibles with infectious individuals at the rate $\beta$. It decreases when they recover from TB due to treatment at the rate $\rho$, TB-induced death at the rate $\delta$ and natural death at the rate $\mu$. Hence

$$
\frac{d I}{d t}=\beta S I-\rho I-\delta I-\mu I
$$

The population of recovered individuals is generated by the recovery of infectious individuals due to treatment at the rate $\rho$. It increases due to wearing of particulate respirator and vaccination of susceptible individuals at the rates $\psi$ and $v$, and decreases due to natural death at the rate $\mu$. Thus

$$
\frac{d R}{d t}=\rho I+\psi S+v S-\mu R
$$

The following assumptions are made in order to formulate the equations of the model

1. Susceptible individuals who comply to particulate respirator move to the recovered compartment

2. Due to short duration of tuberculosis exposed classes, they do not take part in the dynamics.

3. All individuals are born susceptible.

The dynamics of the transmission is represented by the following system of ordinary differential equations:

$$
\begin{aligned}
\frac{d S}{d t} & =\Lambda-\beta S I-v S-\psi S-\mu S \\
\frac{d I}{d t} & =\beta S I-\rho I-\delta I-\mu I \\
\frac{d R}{d t} & =\rho I+\psi S+v S-\mu R
\end{aligned}
$$

with initial conditions $(S(0), I(0), R(0)) \in \Re_{+}^{3} . \Lambda(0)$ is the initial size of individuals that are born or that migrate into the population while $S(0)+I(0)+R(0)=N(0)$ denotes the initial total number of individuals in a population. 
TABLE 1. Summary of the parameters

\begin{tabular}{llll}
\hline Parameter & Meaning & Value & Reference \\
\hline$\mu$ & Natural human death rate & $\left(0.016\right.$ year $^{-1}$ & {$[17]$} \\
$\beta$ & Human-to-human transmission rate & 0.821 day $^{-1}$ & {$[2]$} \\
$\rho$ & Recovery rate due to treatment & 0.5 year $^{-1}$ & Assumed \\
$\Lambda$ & Human recruitment rate & $\left(0.025\right.$ year $^{-1}$ & {$[12]$} \\
$v$ & BCG Vaccination rate & 0.65 year $^{-1}$ & {$[16]$} \\
$\psi$ & compliance rate to particulate respirator by healthcare workers & 0.5 & Assumed \\
$\delta$ & Tuberculosis-induced death rate & 0.1577 & {$[7]$} \\
\hline
\end{tabular}

2.1. Positivity of solutions. For the TB transmission model to be epidemiologically meaningful, it is important to prove that all state variables are non-negative at all time. That is, solution of the system (2.1)-(2.3) with nonnegative initial data will remain non-negative for all time $t>0$.

Theorem 1: Let the initial data be $S(0), I(0), R(0)$ be non-negative. Then the solutions $(S, I, R)$ of $(2.1)-(2.3)$ are positive and bounded for all $t>0$, whenever they exist.

Proof: From (2.1),we have

$$
\frac{d S}{d t}=\Lambda-\beta S I-v S-\psi S-\mu S
$$

This leads to

$$
\frac{d S}{d t} \geq-(\beta I+v+\psi+\mu) S
$$

Using separation of variable, it then follows that

$$
\frac{d S}{S} \geq-(\beta I+v+\psi+\mu) d t
$$

Integrating gives

$$
S(t) \geq S_{o} \exp -\beta \int_{0}^{t} I(\zeta) \zeta-(v+\psi+\mu) t
$$

And we have that

$$
S(t) \geq S_{o} \exp -\beta \int_{0}^{t} I(\zeta) \zeta-(v+\psi+\mu) t>0
$$

if

$$
S_{o}>0
$$

From (2.2), we have

$$
\frac{d I}{d t}=\beta S I-\rho I-\delta I-\mu I
$$

Using separation of variable gives

$$
\frac{d I}{I} \geq-(\rho+\delta+\mu) d t
$$

Integrating gives

$$
I(t) \geq I_{o} \exp -(\rho+\delta+\mu) t
$$

And we have that

$$
I(t) \geq I_{o} \exp -(\rho+\delta+\mu) t>0
$$


if

$$
I_{0}>0
$$

From (2.3),

$$
\frac{d R}{d t}=\rho I+\psi S+v S-\mu R
$$

Thus

$$
\begin{aligned}
& \frac{d R}{d t} \geq-\mu R \\
& \frac{d R}{R} \geq-\mu d t
\end{aligned}
$$

Integration of the above equation yields

$$
\begin{aligned}
& \ln R \geq-\mu t+A \\
& R \geq A \exp -\mu t
\end{aligned}
$$

But

$$
R(0)=R_{o}
$$

And we have that

$$
R \geq R_{o} \exp -\mu t
$$

if

$$
R_{o}>0
$$

This completes the proof

\section{Disease-Free Equilibrium Analysis}

For the equilibrium points, the above model is equated to zero. Thus, we have from (2.1),

$$
S=\frac{\Lambda}{v+\psi+\mu}
$$

and

$I=0$

From (2.3)

$$
R=\frac{v \Lambda}{\mu(v+\psi+\mu)}+\frac{\psi \Lambda}{v(v+\psi+\mu)}
$$

Therefore, the disease-free equilibrium point of the model is

$$
\begin{aligned}
& E_{o}=\left(\frac{\Lambda}{v+\psi+\mu}, 0, \frac{v \Lambda}{\mu(v+\psi+\mu)}+\frac{\psi \Lambda}{v(v+\psi+\mu)}\right) \text { and the unique endemic equilibrium point is } \\
& E_{o}^{*}=\left(S^{*}, I^{*}, R^{*}\right) \text { where } \\
& \qquad S^{*}=\frac{\rho+\delta+\mu}{\beta} \\
& \qquad I^{*}=\frac{\Lambda \beta-(v+\psi+\mu)(\rho+\delta+\mu)}{\beta(\rho+\delta+\mu)}
\end{aligned}
$$


and

$$
R^{*}=\frac{\rho(\Lambda \beta-(v+\psi+\mu)(\rho+\delta+\mu))+(\psi+v)(\rho+\delta+\mu)^{2}}{\mu \beta(\rho+\delta+\mu)}
$$

We next calculate the basic reproduction number which will be used for the establishment of local stability of disease-free and endemic equilibrium solution. This is the average number of secondary infectious individuals generated by a primary case throughout the period of infection in a susceptible population.

Hence we have

$$
R_{o}=\frac{\beta \Lambda}{(v+\psi+\mu)(\rho+\delta+\mu)} .
$$
tion:

The following theorem presents condition that guarantees the local stability of disease-free equilibrium solu-

Theorem 2: The disease-free equilibrium of the model is locally asymptotically stable if $R_{o}<1$ and unstable if $R_{o}>1$

Proof: Consider the equations

$$
\begin{aligned}
& F_{1}=\Lambda-\beta S I-v S-\psi S-\mu S \\
& F_{2}=\beta S I-\rho I-\delta I-\mu I \\
& F_{3}=\rho I+\psi S+v S-\mu R
\end{aligned}
$$

The Jacobian matrix

$$
J_{E}=\left[\begin{array}{ccc} 
& & \\
-\beta I-v-\psi-\mu & -\beta S & 0 \\
\beta I & \beta S-(\rho+\delta+\mu) & 0 \\
\psi+v & \rho & -\mu
\end{array}\right]
$$

The Jacobian of the tuberculosis model (2.1)-(2.3) evaluated at the disease-free equilibrium $E_{o}=\left(\frac{\Lambda}{v+\psi+\mu}, 0, \frac{v \Lambda}{\mu(v+\psi+\mu)}+\frac{v(v)}{v(v)}\right.$ gives

$$
J_{E_{0}}=\left[\begin{array}{ccc}
-(v+\psi+\mu) & -\beta\left(\frac{\Lambda}{v+\psi+\mu}\right) & 0 \\
0 & \beta\left(\frac{\Lambda}{v+\psi+\mu}\right)-(\rho+\delta+\mu) & 0 \\
\psi+v & \rho & -\mu
\end{array}\right]
$$

The eigenvalues of the Jacobian matrix are given by

$$
\begin{aligned}
\lambda_{1} & =-(v+\psi+\mu) \\
\lambda_{2} & =\beta\left(\frac{\Lambda}{v+\psi+\mu}-(\rho+\delta+\mu)\right) \\
& =R_{o}-1 \\
\lambda_{3} & =-\mu
\end{aligned}
$$

and $R_{o}=\frac{\beta \Lambda}{(v+\psi+\mu)(\rho+\delta+\mu)}$

It is easy to see that the eigenvalues have negative real parts if $R_{o}<1$ and thus the disease-free equilibrium point, $E_{o}$, is locally asymptotically stable and unstable for $R_{o}>1$. The epidemiological implication of the above theorem is that tuberculosis will die out of the population if the initial size of the infectious population is small enough (i.e it is in the basin of attraction for disease-free equilibrium points). 
Also, it is observed from theorem (2) that whenever $R_{o}>1$, the asymptotic local stability of the disease-free equilibrium is lost. So if $R_{o}=1$, then the asymptotic local stability of the disease-free equilibrium is exchanged for asymptotic local stability of the endemic equilibrium. This can be explored through a center manifold theory of bifurcation analysis described in ([1]). The epidemiological implication of $R_{o}=1$ is that malaria does not die out of the population and it is not also spreading because for most diseases, we just want to stop their spread.

Hint:

For polynomials of degree $2,3,4,5$, the Routh-Hurwitz criteria are summarized as follows:

$n=2: a_{1}>0$ and $a_{2}>0$

$n=3: a_{1}>0, a_{3}>0$ and $a_{1} a_{2}>a_{3}$

$n=4: a_{1}>0, a_{3}>0, a_{4}>0$ and $a_{1} a_{2} a_{3}>a_{3}^{2}+a_{1}^{2} a_{4}>0$

$n=5: a_{1}>0, i=1,2,3,4,5, a_{1} a_{2} a_{3}>a_{3}^{2}+a_{1}^{2} a_{4}>0$ and $\left(a_{1} a_{4}-a_{5}\right)\left(a_{1} a_{2} a_{3}-a_{3}^{2}-a_{1}^{2} a_{4}\right)>a_{5}\left(a_{1} a_{2}-a_{3}\right)^{2}+a_{1} a_{5}^{2}$

\section{Endemic Equilibrium Analysis}

We have the following theorem on the local asymptotic stability of the endemic equilibrium $E_{0}^{*}$ :

Theorem 3: The endemic equilibrium of the model is locally asymptotically stable if $R_{o}>1$

Proof: Consider the equations

$$
\begin{aligned}
& F_{1}=\Lambda-\beta S I-v S-\psi S-\mu S \\
& F_{2}=\beta S I-\rho I-\delta I-\mu I \\
& F_{3}=\rho I+\psi S+v S-\mu R
\end{aligned}
$$

Linearizing the system at the endemic equilibrium point $E_{o}^{*}=\left(S^{*}, I^{*}, R^{*}\right)$ gives

$$
J_{E_{o}}^{*}=\left[\begin{array}{ccc} 
& & \\
-\beta I^{*}-v-\psi-\mu & -\beta S^{*} & 0 \\
\beta I^{*} & \beta S^{*}-(\rho+\delta+\mu) & 0 \\
\psi+v & \rho & -\mu
\end{array}\right]
$$

Definition: Let $J_{E_{0}}$ be any square matrix of order $\mathrm{n}$. The characteristic equation of $J_{E_{0}}$ is $\left|J_{E_{0}}-\lambda I\right|=0$

A necessary and sufficient condition for local asymptotic stability is for the real part of the eigenvalue to be in the negative half plane. Thus, we need to show that $J_{E_{o}}^{*}$ given by (4.4) has all its eigenvalues with negative real part. In what follows, the characteristic equation of $J_{E_{o}}^{*}$ is of the form

$$
\lambda^{3}+a_{1} \lambda^{2}+a_{2} \lambda+a_{3}=0
$$

where

$$
\begin{aligned}
& a_{1}=\mu(\mu+\rho+\delta)+\Lambda \beta \\
& a_{2}=\Lambda \beta-(v+\psi+\mu)(\rho+\delta+\mu)+\frac{\Lambda \beta \mu}{\rho+\delta+\mu} \\
& a_{3}=\Lambda \beta \mu-\mu(v+\psi+\mu)(\rho+\delta+\mu)
\end{aligned}
$$

Clearly, $a_{1}>0 . a_{2}>0$ since $a_{1}>0$. Also, $a_{3}$ can be written in terms of $R_{o}$ as

$$
a_{3}=\mu(\mu+v+\psi)(\rho+\delta+\mu) R_{o}-1
$$

If in (4.6), $R_{o}>1$, then $a_{3}>0$. Since the coefficients $a_{i}, i=1,2,3$ and the Hurwitz matrices of the polynomial (4.5) are positive, using Routh-Hurwitz criterion (see[8]), all the eigenvalues of (4.5) have positive real parts. By Routh-Hurwitz conditions, i.e., $a_{1}>0, a_{3}>0$ and $a_{1} a_{2}>0$. Then the endemic equilibrium, $E_{0}^{*}$, is stable. The epidemiological implication of this, is that tuberculosis establishes itself in the population for $R_{0}>1$. 


\section{Sensitivity Analysis}

We conducted a sensitivity analysis of parameters of the model system (2.1) - (2.3) in order to determine the relative importance of the model parameters on the disease infection. To determine how best to reduce the infection, it is necessary to know the relative importance of the different factors responsible for the infections.

Sensitivity indices could be computed numerically so as to figure out parameters that have high impact on basic reproduction number $R_{0}$ and which of the parameters should be given preferential treatment by intervention strategies.

Analytically, sensitivity analysis on all parameters which account for disease dynamics is done using Chitnis et al (2008) approach, we compute sensitivity indices of the $R_{0}$ which measures initial disease infection and allow us to measure relative change in a state variable when a variable changes.

The normalized forward sensitivity index of a variable to a parameter is the ratio of the relative change in the variable to the relative change in the parameter. When the variable is a differentiable function of the parameter, the sensitivity index may be alternatively defined using partial derivatives.

Definition The normalized forward sensitivity index of a variable, $u$, that depends differentiably on a parameter, $\mathrm{p}$, is defined as:

for $\mathrm{u} \neq 0$

$$
N_{p}^{u}=\frac{\partial u}{\partial p} \times \frac{p}{u}
$$

Consequently, we derive analytical expression for the sensitivity index of $R_{0}$ as

$$
N_{p_{i}}^{R_{0}}=\frac{\partial R_{0}}{\partial p_{i}} \times \frac{p_{i}}{R_{0}}
$$

where $p_{i}, i \in \mathbb{N}$ denotes each parameter involved in $R_{0}$

Using

$$
R_{o}=\frac{\beta \Lambda}{(v+\psi+\mu)(\rho+\delta+\mu)}
$$

and parameters values in Table 1 . We compute sensitivity index of each parameter with respect to the $R_{0}$, for instance:

$$
N_{\beta}^{R_{0}}=\frac{\partial R_{0}}{\partial \beta} \times \frac{\beta}{R_{0}}=0.03183 \times 31.577=1.0051
$$

We have Table 2 which summarizes the sensitivity indices of $R_{0}$ with respect to parameters

$$
N_{\Lambda}^{R_{0}}, N_{v}^{R_{0}}, N_{\psi}^{R_{0}}, N_{\mu}^{R_{0}}, N_{\delta}^{R_{0}}, N_{\rho}^{R_{0}}
$$

The sign of the sensitivity indices of $R_{0}$ with respect to most of the parameters agree with intuitive expectation. Keeping all others factors constant, we observe that most sensitive parameter is $\beta$ (Human-to-human transmission rate) followed by $\Lambda$ (Human recruitment rate). Since $N_{\beta}^{R_{0}}=+1.0051$, it implies increase in $\beta$ tends to increase $R_{0}$. Similarly, $N_{\Lambda}^{R_{0}}=+1.004$ indicates positive linear change in $\Lambda$ with respect to $R_{0}$. On the contrary, increase in any other parameters decreases $R_{0}$ since $N_{v}^{R_{0}}, N_{\psi}^{R_{0}}, N_{\mu}^{R_{0}}, N_{\delta}^{R_{0}}, N_{\rho}^{R_{0}}$ are all negative. Though the effect of the decrease will be determined by the sensitivity index.

\section{Interpretation of sensitivity Indices obtained in Table 2}

In other words, we mean that basic reproduction number $R_{0}$ is directly proportional to $\beta$ and $\Lambda$ and inversely proportional to other parameter. Specifically, increase in $\psi$ (compliance rate to particulate respirator by healthcare 
TABLE 2. Numerical values of sensitivity indices of $R_{0}$ with respect to parameter involved.

\begin{tabular}{|c||c|}
\hline \hline Parameter symbol & Sensitivity Index \\
\hline \hline$\beta$ & +1.0051 \\
\hline$\Lambda$ & +1.004 \\
\hline$v$ & -0.5591 \\
\hline$\psi$ & -0.43 \\
\hline$\mu$ & -0.0377 \\
\hline$\delta$ & -0.2352 \\
\hline$\rho$ & -0.7459 \\
\hline
\end{tabular}

workers) leads to decrease in $R_{0}$ which determines how the disease infection goes into extinction. Finally, we deduce that Human-to-human transmission rate and Human recruitment rate are very sensitive to number of secondary infections caused by single one.

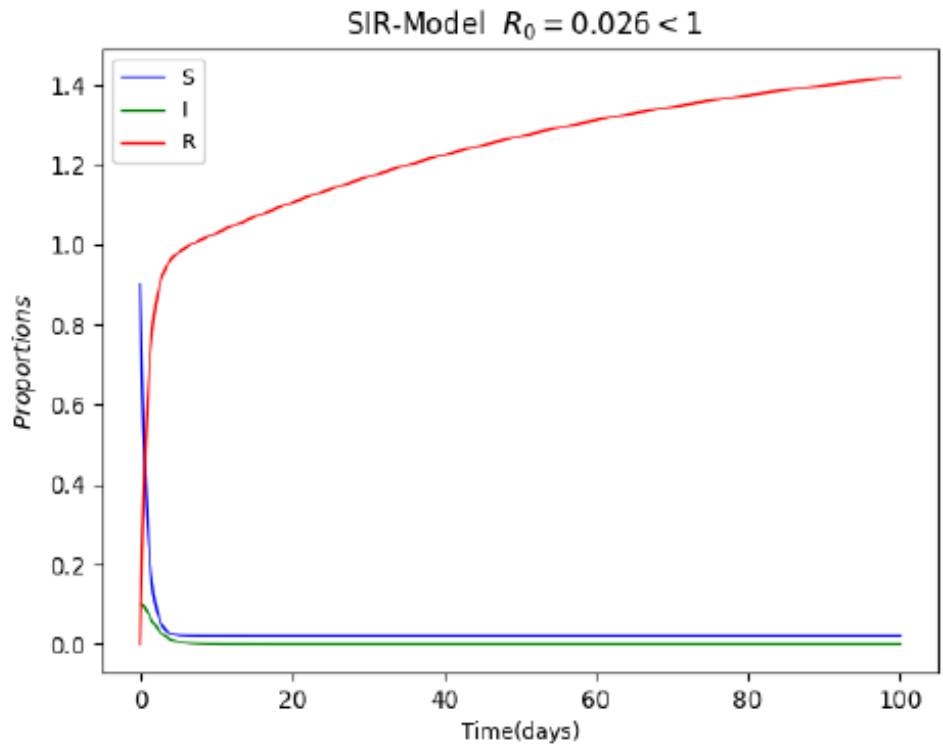

Figure 1: Parameters value: $\beta=0.821, \Lambda=0.025, v=0.65$, $\psi=0.5, \mu=0.016, \rho=0.5, \delta=0.1577, S(0)=0.9, I(0)=0.1$, $R(0)=0.0$

Figure 1. Parameters value: $\beta=0.821, \Lambda=0.025, v=0.65, \psi=0.5, \mu=0.016$, $\rho(T)=0.5, \delta=0.1577, S(0)=0.9, I(0)=0.1, R(0)=0.0($ from Table 1$)$ 


\section{Numerical simulation}

In this section, we carried out simulation showing dynamics of the disease in the population using Python(Canopy) program. The colors distinguish the various steady state variables in the model.

\section{Interpretation of Figure 1}

Figure 1 shows the dynamics of the model system (2.1) - (2.3). At $R_{0}=0.026<1$, we observe that the proportion of the susceptible population (colored blue) declines, so as the Infected population (colored green) which goes to extinction completely within few days. Meanwhile, the proportion of recovery population(colored red) increases logarithmically during the process. The implication is that there is stability in dynamical system of the model at $R_{0}<1$.

NOTE: We have chosen to use different color in Figure 1, not only to distinguish the steady state variables but also for clarity and brevity since usage of similar color(e.g black) for the steady state variables will make it difficult to comprehend the interpretation.

\section{Conclusion}

In this research work, a mathematical model with particulate respirator compliance is developed and analysed to study the transmission and control of Tuberculosis. We first showed that the solution of (2.1)-(2.3) are positive and bounded for all $t>0$ whenever they exist. The model incorporates control measure $\psi$, which is the rate at which healthcare workers comply to wearing particulate respirator each time they are in close contact with TB patients or when they are in the environment where TB patients are present. Disease-free and endemic equilibrium of the model were obtained and analysed for stability. We also obtained $R_{o}$. It is known that when $R_{o}<1$, TB dies out of the population and when $R_{o}>1$, TB persists in the population. In addition, we carried out sensitivity analysis to examine the factors most responsible for the transmission and spread of TB among healthcare workers. Numerical simulation was also performed to understand the overall picture of the disease behaviour.

\section{Contributions to Knowledge}

- A new parameter $\psi$, which is the compliance rate to particulate respirator by healthcare workers, was introduced into tuberculosis model. This parameter has not been considered by the existing tuberculosis models.

- Public healthcare workers can educate people on the effect of the incorporated parameter(compliance rate to particulate respirator) on transmission dynamics of tuberculosis model by using this article as a study guide for seminars, workshop, or training programs.

- Sensitivity analysis of the extended model was carried out to know how large the parameter affects the basic reproduction number $R_{o}$ negatively. Therefore, the result of the analyses can be useful in public health to know which factor decreases or increases $R_{0}$, so that intervention strategies can be done to reduce tuberculosis transmission.

\section{Bibliography}

1. C. Castillo-Chavez, B. Song, Dynamic models of tuberculosis and their applications, Mathematical Biosciences and Engineering, 1(2), 361-404. 2004.

2. R. Diel, R. Loddenkemper, S. Niemann and P.R. Meywald-Walter Narayanan, Restriction fragment length polymorphism typing of clinical isolates of my mycobacterium tuberculosis from patients with pulmonary tuberculosis in Madras, South India. Tuber Lung Dis, 76(6), 550-554. 1995.

3. D.M.Dago, M.O. Ibrahim and A.S. Tosin, Stability analysis of a deterministic mathematical model for transmission dynamics of tuberculosis. International Journal of Advances in Science Engineering and Technology, ISSN, 2, 
2321-9009. 2015.

4. Department of Health of Indonesia, Prevention of Tuberculosis Handbook, 2008.

5. K.Q. Fredlina, T.B. Oka and I.M. Dwipayana, SIR(Susceptible, Infectious, Recovered) Model for Tuberculosis Disease Transmission, J. Matematika 1(1), 52-58. 2012.

6. N.N. Leontine, T.M. Thomas, A. Franklin and M.M. Martin Luther, Mathematical model to assess vaccination and effective contact rate impact in the spread of tuberculosis, Journal of Biological Dynamics, 13(1). 2019.

7. D.P. Moualeu, S. Bowong and J. Kurths, Parameter estimation of a tuberculosis model in a patch environment: case of Cameroon, Proc. Int. Sym. Math. Comput. Biol., 5. 352-373.

8. J.D. Murray, Mathematical Biology I, An introduction, 3rd ed. Springer, Heidelberg, 2001.

9. J. Nainggolan, S. Sudradjat, A.K. Supriatna and N. Anggriani, Mathematical model of tuberculosis, Journal of Physics: Conference Series, 423. 2013.

10. L. Prihutami, Stability analysis of tuberculosis transmission model, Thesis, Diponegoro University, Semarang, 2009.

11. T. Iskandar, N. Ayningtia, S. Munzir, V, Halfiani and M. Ramli, Mathematical model of tuberculosis epidemic with recovery time delay, Department of Mathematics, Syiah Kuala University, Banda Aceh, Indonesia, 2311. 2017.

12. United Nations Department of Economic and Social Affairs/Population Division, World Population Prospects, 2. 1-4. 2012.

13. V. K. Gupta, S.k. Tiwari, S. Sharma and L. Nagar, Mathematical model of tuberculosis with drug resistance to the first and second line of treatment, Journal of New Theory, 1. 94-106. 2018.

14. WHO policy on TB infection control in health care facilities, Congregate Settings and Households Geneva, World Health Organization, 2009.

15. World Health Organization (WHO), Global Tuberculosis Report, 2016.

16. World Bank, World Development Indicators, 2013.

17. World Health Organization, Global health observatory data respiratory. Life expectancy: Life expectancy by country, World Health Organization, Geneva, Switzerland, 2013.

18. Y. Yu, Y. Shi and W. Yao, Dynamic model of tuberculosis considering multi-drug resistance and their applications, 8 2. 362-372. 2018. 